\title{
Rapid Detection and Treatment of Inborn Errors of Metabolism in the Newborn Period: Beginning of a New Trend
}

\author{
Sunita Bijarnia-Mahay ${ }^{1}$ (D) $\cdot$ Ranjana Mishra $^{1}$
}

Received: 3 March 2021 / Accepted: 15 March 2021 / Published online: 26 March 2021

(C) Dr. K C Chaudhuri Foundation 2021

Inborn errors of metabolism (IEMs) are a heterogeneous group of genetic and biochemically determined disorders, classically manifesting early in life. A large percentage of these neonatally presenting disorders are treatable, thus making them amenable to newborn screening. Newborn screening (NBS) is still in the nascent stage in our country with $<3 \%$ of the total newborn population being screened for at least one disorder [1]. Thus, majority of the centers are still diagnosing the IEMs after the clinical symptoms have appeared. Nonetheless, the optimism is still intact since more and more babies are now being recognized in the newborn period, setting a trend made possible by availability of advanced tests such as tandem mass spectrometry as well as genomic testing via next generation sequencing (NGS).

The turn of the century marked the dawn of a new era of genomics, which would pave the way for the then impossible treatments of genetic disorders. The successful culmination of the Human Genome Project ushered in an exciting era of improved diagnostics, aided by technology, translating into the possibilities of genetic therapies in the next decade. We are now in the era of treatment of genetic disorders. Inborn errors of metabolism have shown the way, being the largest group of 'treatable' genetic disorders [2]. India is rising up to the occasion, albeit in smaller scale. We are fast catching up with the technology to diagnose more disorders. As newborn screening has established itself as one of the most successful preventive health programs, we are starting to embrace it, little by little. Spearheaded by the ICMR task force, many states have initiated the NBS program including the state of Kerala $[1,3]$. The article by Balakrishnan et al. is refreshing and provides a glimpse of the profile of inborn errors of

Sunita Bijarnia-Mahay

bijarnia@gmail.com

1 Institute of Medical Genetics \& Genomics, Sir Ganga Ram Hospital, New Delhi 110060, India metabolism in South India, detected by a combination of newborn screening (NBS, healthy babies) and those that were symptomatically detected (sick babies), after manifesting symptoms [4]. Balakrishnan et al., in their study, present a cohort of 33 newborns that were diagnosed with an IEM, and provide a follow-up for up to 12 mo of age. Among the 33 babies, 3 were diagnosed by NBS; 16 died either in neonatal period or later, 14 survived till 12 mo of age, and 3 were lost to follow-up.

There are several takeaway points from this study. The first is the incidence of group of IEMs amenable to treatment in the newborn screened population. In the cohort of 18,720 newborns, only 3180 babies underwent extended NBS to include the IEMs, and 3 babies were detected, providing an incidence of such disorders to be 1 in 1060. Although the sample size is much too small, it is still notable and perhaps giving a glimpse of what is to follow in large-scale studies. It is possible that consanguinity may have been a factor, since $64 \%$ of the diagnosed newborn population belonged to consanguineous families. Second point from the study is the evidence that NBS saves lives! In the cohort of 16 nonsurvivors, none had undergone NBS, as compared to 3 of 14 survivors who were detected via NBS. All the three babies [1 medium-chain acyl-coenzyme A dehydrogenase deficiency (MCADD) and 2 biotinidase deficiencies] were alive and well, saved from their potentially life-threatening condition. The other factor which is evident here, apart from the disease severity would be the 'timing' of diagnosis. The earlier the detection, the better is the prognosis, as was the case with babies with citrullinemia, type 1 and fructose-1,6-bisphosphatase deficiency.

The third notable point was that genomic testing did not play any significant role in the detection of these IEMs. In the current wave of genomic testing, at least in India where NGSbased genomic testing is fancied and considered the 'gold standard' for all genetic disorders, this again seals the fact that biochemical testing shall remain fundamental in diagnosing patients with IEMs. The role of NGS-based genomic testing in this particular group of IEMs lies in the confirmation of 
doubtful cases, and enabling carrier screening and prenatal diagnosis in at-risk couples in the family. Lastly, with improved survival of babies with IEMs such as maple syrup urine disease (MSUD) and propionic acidemia, it is heartening to see that the combined efforts of specialists, parent organizations, and the government is bearing fruit in making the treatments of IEMs, especially dietary management accessible to the average Indian [5]. In the authors' personal experience, this is gradually changing the natural course of the disease in a positive way.

\section{Declarations}

Conflict of Interest None.

\section{References}

1. Mookken T. Universal implementation of newborn screening in India. Int J Neonatal Screen. 2020;6(2):24. https://doi.org/10.3390/ ijns6020024.
2. van Karnebeek CD, Stockler-Ipsiroglu S. Early identification of treatable inborn errors of metabolism in children with intellectual disability: the treatable intellectual disability endeavor protocol in British Columbia. Paediatr Child Health. 2014;19(9):469-71. https://doi.org/10.1093/pch/19.9.469.

3. ICMR Task Force on Inherited Metabolic Disorders. Newborn screening for congenital hypothyroidism and congenital adrenal hyperplasia. Indian J Pediatr. 2018;85:935-40. https://doi.org/10.1007/ s12098-018-2645-9.

4. Balakrishnan U, Chandrasekaran A, Amboiram P, Ninan B, Ignatious $\mathrm{S}$. Outcome of inherited metabolic disorders presenting in the neonatal period. Indian J Pediatr. 2020. https://doi.org/10. 1007/s12098-020-03522-6.

5. Bijarnia-Mahay S, Verma IC. Inborn errors of metabolism in Indiawhere we are at! Indian J Pediatr. 2019;86(10):875-6. https://doi. org/10.1007/s12098-019-03067-3 Epub 2019 Sep 10.

Publisher's Note Springer Nature remains neutral with regard to jurisdictional claims in published maps and institutional affiliations. 